\title{
A Many-objective Particle Swarm Optimization Algorithm Based on Multiple Criteria for Hybrid Recommendation System
}

\author{
Zhaomin Hu, Yang Lan, Zhixia Zhang, and Xingjuan Cai* \\ Taiyuan University of Science and Technology, School of Computer Science and Technology \\ Taiyuan, Shanxi 030024, China \\ [e-mail: Hzming11@163.com, \{lanyangvip1020, 15634969919, xingjuancai\}@ 163.com] \\ ${ }^{*}$ Corresponding author: Xingjuan Cai \\ Received December 30, 2020; revised January 23, 2021; accepted February 1, 2021; \\ published February 28, 2021
}

\begin{abstract}
Nowadays, recommendation systems (RSs) are applied to all aspects of online life. In order to overcome the problem that individuals who do not meet the constraints need to be regenerated when the many-objective evolutionary algorithm (MaOEA) solves the hybrid recommendation model, this paper proposes a many-objective particle swarm optimization algorithm based on multiple criteria (MaPSO-MC). A generation-based fitness evaluation strategy with diversity enhancement (GBFE-DE) and ISDE $^{+}$are coupled to comprehensively evaluate individual performance. At the same time, according to the characteristics of the model, the regional optimization has an impact on the individual update, and a many-objective evolutionary strategy based on bacterial foraging (MaBF) is used to improve the algorithm search speed. Experimental results prove that this algorithm has excellent convergence and diversity, and can produce accurate, diverse, novel and high coverage recommendations when solving recommendation models.
\end{abstract}

Keywords: Many-objective Particle Swarm Optimization Algorithm, Recommendation System, Fitness Estimation Method, Internet of Things 


\section{Introduction}

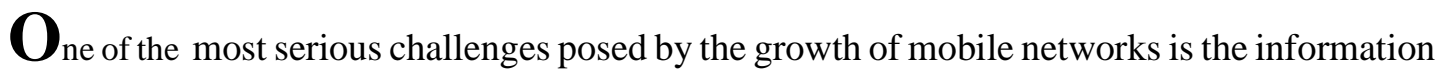
overload caused by massive amounts of data. Recommendation system (RS) [1] as a technology that automatically processes enormous amounts of data to mine user preference can effectively deal with the problem of information overload. Up till the present moment, RS is used in various areas of life, including entertainment [2-4], shopping [5], learning [4], travel [6], and so on.

Traditional RSs [7] only pay close attention to how to provide accurate recommendation to users $[8,9]$. With the increase of user demand, it becomes more and more significant to provide users with recommendations that include a variety of excellent performance [10]. In recent years, some researchers have begun to optimize multiple recommended performances. Most of these studies optimize the accuracy and diversity of recommendations to provide users with recommendation lists that meet user preferences and attract users' continuous attention. Geng [11] provided users with a set of candidate items through collaborative filtering (CF) [12] and then used NNIA to combine recommendation lists. Zuo [13] adopted a variety of different MaOEA to optimize the items in the Top-N recommendation list. Nevertheless, novelty [10] can recommend long-tail items (non-popular items) as much as possible, and coverage can make as many items in the system recommended as possible, which are all essential indicators in the RS [14]. Therefore, some models that can simultaneously optimize recommendation accuracy, diversity, novelty, and coverage are put forward as many-objective optimization problem (MaOP) [15].

Intelligent optimization algorithms are divided into three categories according to the number of optimization objectives: single evolutionary algorithm [16-18], multi-objective evolutionary algorithm (MOEA) [19] and MaOEA [20-22]. The increase of optimization objectives will lead to a large number of non-dominated solutions during the algorithm execution process [23]. Therefore, the convergence and diversity of the algorithm cannot be well balanced when the strategy of MOEA is used to deal with the MaOPs, which results in the population cannot move rapidly towards the Pareto-optimal Front (PF). As a consequence, intelligent optimization algorithms that optimize problems containing more than 4 objectives are termed as MaOEA.

Most of the existing research on MaOEA focus on the algorithm mechanism based on evolutionary behavior. The purpose of these mechanisms is to enhance the pressure of environmental selection by designing appropriate environmental selection strategies to make individuals move towards PF. NSGA-III [24] set up uniformly distributed reference points in the decision space and combined non-dominated sorting strategies to maintain the convergence and diversity of the algorithm. RVEA [25] proposed the concept of reference vector and selects individuals by judging the angle of individuals and reference vectors. Yang [26] proposed a grid-based evolutionary algorithm (GrEA), which uses grid-based evaluation metrics to enhance selection pressure, to solve MaOPs. However, this kind of algorithm generates the offspring through crossover and mutation operators (such as simulated binary crossover), and there is no direct correlation between the individual in current generation and the previous generation. The MaOEA based on swarm intelligence optimization makes the individuals in the population move to achieve the function of updating the individual. These methods can keep the historical information and individuality of the solution in the population. NMPSO [27], which belong to many-objective particle swarm optimizers (MaPSO) [28-30], proposed a novel BFE to balance the convergence and diversity of individuals in the population. 
Due to MaOEA based on swarm intelligence optimization needs to select excellent individuals in the population, the remaining individuals move towards the direction of the excellent individuals. The judgment of excellent individuals is affected by individual convergence and diversity, which also determines the performance of the algorithm. The archive strategy is introduced to store the excellent individuals in the population, but the archive strategy with a single individual evaluation criterion results in the algorithm performance being determined only by the selected evaluation criterion. Commonly used evaluation criteria include: HV [31, 32], IGD [33], GD, etc. HV is used to calculate hypervolume to comprehensively evaluate the convergence and diversity of individuals, but it requires a lot of computing power. Cai [34] adopted a generation-based fitness evaluation (GBFE) criterion, which emphasizes convergence in the early stage of the algorithm and diversity in the later stage of the algorithm. On this basis, an archiving strategy containing multiple criteria can effectively solve this problem, and the results of the algorithm can be improved from multiple perspectives through the coupling of multiple evaluation criteria.

For the research of many-objective optimization recommendation algorithm, Cai introduced GBFE and partition-based knowledge mining strategy based on NSGA-III. When NSGA-III as a MaOEA based on evolutionary behavior [35] solves the constraint problem, there are a large number of individuals who do not meet the constraint in the offspring generated by the parent. Although there are corresponding constraint processing strategies, it greatly affects the performance of the algorithm. However, the MaOEA based on swarm intelligence optimization [36] records the individual personal-best position and global-best position of each solution in the population. Even if the individual update in a position beyond the constraint range, it can be replaced by the individual personal-best position and global-best position. Therefore, MaPSO-MC is proposed to deal with the DTLZ test suite and hybrid recommendation model. Our contributions are summarized as follows:

1) For traditional MaPSO, a single individual evaluation strategy is used to select archive. We present a MaPSO-MC, which uses GBFE-DE and $\operatorname{ISDE}^{+}$[37] as individual evaluation criteria to choose outstanding individuals in population from multiple perspectives.

2) To solve the hybrid RS with MaOEA, partition-based particle swarm updating strategy (PBPS) and MaBF are proposed. During the mating process, the individual will be affected by the personal-best, global best and regional-best. Meanwhile, superior individuals will be copy through MaBF in late-stage of algorithm.

3) Aiming at the problem that the proportion of diversity in the early-stage individual evaluation of the algorithm in the generation-based fitness evaluation strategy is too small. A GBFE-DE is proposed to enhance the diversity of algorithm and prevent the algorithm from falling into a local optimum in the early stage.

The remainder of this paper is organized as follows. Section 2 introductions the background including hybrid recommendation model and the many-objectives particle swarm optimization algorithm. In Section 3, we describe in detail the many-objective particle swarm optimization algorithm based on multiple criteria. Section 4 shows the mechanisms of GBFEDE strategy and PBPS strategy and their applications in MaOEAs in details. In Section 5, we show the settings and results of the experiment. Finally, the conclusion is given in Section 6. 


\section{Background}

\subsection{Hybrid Recommendation Model Based on MaOEA}

Since user's demand for recommendation performance is not limited to accuracy and diversity, and recommendation results directly affects the profit of merchants. The hybrid recommendation model based on MaOEA proposed by Cai [34] can concurrently optimize the accuracy, recall, diversity, novelty, and coverage.

Hybrid model can be applied to most recommendation scenarios through linear combination of item-based CF, user-based CF, and hybrid recommendation techniques by content-based and CF. The formula is shown in (1):

$$
R_{f i n}=\alpha \times R_{U-c f}+\beta \times R_{I-c f}+\gamma \times R_{h y b}
$$

where $\alpha, \beta$, and $\gamma$ respectively denote the combination coefficients of the three basic techniques. Therefore, there are the following constraints:

$$
1=\alpha+\beta+\gamma
$$

Moreover, in order to reduce the number of decision variables to improve the optimization speed, $\alpha$ and $\beta$ are used as decision variables, and $\gamma$ is obtained by (3):

$$
\gamma=1-\alpha-\beta, \quad \alpha+\beta \leq 1
$$

MaOEA is used to train the parameters that combine the three basic algorithms to make the predicted rating fit closer to the actual rating.

Accuracy, recall, diversity, novelty, and coverage are used as optimization objectives of the hybrid model to improve the performance of recommendation. The accuracy and recall are calculated by the precision and recall in the confusion matrix of the classification problem. Diversity describes the average similarity between items in the recommendation list. The novelty is used to calculate the proportion of non-popular items in the recommendation list. The coverage is the proportion of recommended items to the total items in the entire system.

\subsection{Many-objective particle swarm optimizers}

The essence of problems in real life are MaOPs, which are defined as follow:

$$
\min F(x)=\left(f_{1}(x), f_{2}(x), \ldots, f_{n}(x)\right) \quad n \geq 4
$$

where $x=\left(x_{1}, x_{2}, \ldots, x_{m}\right) \in \Omega$ is $\mathrm{n}$-dimension decision variable. $\Omega$ and $F(x) \in R^{n}$ are decision space and objective space. MaOEA has distinguished performance when solving such problems. MaPSO is a common method in MaOEA based on swarm intelligence optimization.

MaPSO is to design a many-objective optimization strategy based on the individual location update strategy of single-objective particle swarm optimization algorithm. MaPSO is mainly divided into Pareto-based MaPSO and decomposition-based MaPSO. However, their core idea is to judge the optimal solution in the population, so that the update of the individuals in the population is affected by the global-best and the personal-best. The speed update formula of individual $i$ is as follows: 


$$
v_{i}(t+1)=w v_{i}(t)+c_{1} r_{1}\left(x_{\text {pbest }}-x_{i}(t)\right)+c_{2} r_{2}\left(x_{\text {gbest }}-x_{i}(t)\right)
$$

where $t$ is the generation number; $w, c$, and $r$ are the inertia weight, learning factors and a random number between 0 and 1 , respectively; $x_{\text {pbest }}$ and $x_{g b e s t_{i}}$ respectively represent the personal-best position and global-best position of individual $i$. The individual position is update as shown in (6):

$$
x_{i}(t+1)=x_{i}(t)+v_{i}(t+1)
$$

In MaOPs, the quality of an individual is often affected by multiple evaluation indicators. Therefore, in MaPSO, the global-best often contains many solutions, namely archive, for storing the optimal solutions in the population.

\section{Many-objective Particle Swarm Optimization Algorithm based on Multiple Criteria}

\subsection{The Multiple Criteria}

In MaPSO, individual evaluation criteria will directly affect the performance of the algorithm. Therefore, multiple criteria (GBFE-DE and ISDE ${ }^{+}$) are used to select excellent individuals for archive to optimize individuals in the population from different perspectives. GBFE-DE pays attention to the influence of algorithm generation when evaluating individuals, so that the algorithm focuses on individual convergence in the early stage and diversity in the later stage. $\mathrm{ISDE}^{+}$, as a criterion for comprehensively assessing individual convergence and diversity, evaluates individual performance by calculating individual objective function sequence and SDE.

A. Generation-based Fitness Evaluation with Diversity Enhancement

In MaOEA, individual evaluation is often affected by its convergence and diversity. In order to comprehensively evaluate the convergence and diversity of individuals and overcome the problem of the small proportion of individual diversity in the early stage of the standard GBFE strategy, this paper proposes a GBFE-DE strategy. In this strategy, when evaluating individuals, the early stage of the algorithm is biased toward the convergence of the individuals, and the individuals in the population move toward the PF. On the contrary, the diversity of the individual evaluation in the later stage of the algorithm is more important, so that the individuals in the population are distributed as evenly as possible. The GBFE-DE calculation formula of individual $i$ in population $P$ is as follows:

$$
\text { GBFE }-D E_{i}=\left(1+\left(M \cdot\left(\frac{t}{t_{\max }}\right)^{\theta}+N(0.05,1)\right) \cdot \text { Div }_{i}\right) \cdot\left(1-\text { Con }_{i}\right)
$$

where $M$ is the number of objectives; $t$ and $t_{\max }$ represent the current generation and maximum generation of algorithm; $\mathrm{Div}_{i}$ and $\mathrm{Con}_{i}$ denote the diversity and convergence of individual $i ; N(0.05,1)$ is gaussian distribution.

The diversity $D i v_{i}$ of individual $i$ is determined by its shift-based density estimation 
(SDE) value. It is shown in (8):

$$
D i v_{i}=\frac{S D E_{i}-S D E_{\min }}{S D E_{\max }-S D E_{\min }}
$$

where $S D E_{\max }$ and $S D E_{\min }$ are the maximum and minimum $S D E$ of all individuals in the population. The $S D E_{i}$ for individual $i$ is shown as follow:

$$
\begin{gathered}
S D E_{i}=\min _{p_{i, j} \in P, j \neq i} \sqrt{\sum_{k=1}^{m} \operatorname{sde}\left(f_{k}^{\prime}\left(p_{i}\right), f_{k}^{\prime}\left(p_{j}\right)\right)^{2}} \\
\operatorname{sde}\left(f_{k}^{\prime}\left(p_{i}\right), f_{k}^{\prime}\left(p_{j}\right)\right)=\left\{\begin{array}{cl}
f_{k}^{\prime}\left(p_{j}\right)-f_{k}^{\prime}\left(p_{i}\right) & \text { if } f_{k}^{\prime}\left(p_{j}\right)>f_{k}^{\prime}\left(p_{i}\right) \\
0 & \text { otherwise }
\end{array}\right.
\end{gathered}
$$

$f_{k}^{\prime}\left(p_{i}\right)$ is the normalized result of individual $p_{i}$ in the population $P$ on the k-th objective:

$$
f_{k}^{\prime}\left(p_{i}\right)=\frac{f_{k}\left(p_{i}\right)-f_{k \min }}{f_{k \max }-f_{k \min }}
$$

where $f_{k \max }$ and $f_{k \min }$ are the maximum and minimum value of kth objective in the population. The convergence $C_{i}$ of individual $i$ is calculate by (12):

$$
\operatorname{Con}_{i}=\frac{\operatorname{Dis}\left(i, z^{*}\right)}{\sqrt{m}}
$$

where $z^{*}$ are the ideal point; $\operatorname{Dis}\left(i, z^{*}\right)$ represent the distance between the individual I and ideal point. In addition, since all individuals in the population need to be normalized on each objective during the running of the algorithm, the $\operatorname{Dis}\left(i, z^{*}\right)$ is calculated as follows:

$$
\operatorname{Dis}\left(i, z^{*}\right)=\sqrt{\sum_{k=1}^{m} f_{i, k}^{\prime 2}}
$$

In the early stage, the coefficient of diversity is small, and individual convergence directly affects the quality of individuals; in the later stage of the algorithm, individuals in the population converge to the $\mathrm{PF}$, and the individual convergence indicators are not much different, and diversity mainly determines the individual's GBFE-DE value.

\section{B. Individual Shift-based Density Estimation}

As an indicator to evaluate diversity, SDE considers the influence of convergence in its calculation. The relative density relationship between individuals is judged by shifting individual positions, and it can be calculated as shown in (9) and (10).

Since SDE is an evaluation indicator based on diversity, ISDE+ adds sum of objectives (SB) strategy to SDE to enhance the proportion of individual convergence. The individual SB 
value is obtained by normalizing and summing its multiple objective values, as follows:

$$
S B_{i}=\sum_{k=1}^{m} f_{k}^{\prime}
$$

Individuals that affect the ISDE+ value of an individual $i$ are these in the population whose SB value is better than it. It can be calculated by (15):

$$
I S D E_{i}^{+}=\min _{p_{j} \in P_{S B\left(p_{i}\right), p_{i} \neq p_{j}}} \sqrt{\sum_{k=1}^{m} \operatorname{sde}\left(f_{k}^{\prime}\left(p_{i}\right), f_{k}^{\prime}\left(p_{j}\right)\right)^{2}}
$$

where $p_{i} \in P$ and $p_{j} \in P_{S B\left(p_{i}\right)}$. Meanwhile, $S B\left(p_{i}\right)>S B\left(p_{j}\right)$. A larger ISDE+ means that the individual $i$ has better performance.

\subsection{The Structure of MaPSO-MC}

In the MaOP, individual evaluation cannot use the objective function to directly select outstanding individuals. MaPSO-MC uses multiple different evaluation criteria to select personal-best position and global-best position. At the same time, multiple objectives will result in the global-best position containing multiple individuals instead of one individual. Therefore, the archive strategy is used to store individuals in the global-best position. The pseudo code of Algorithm 1 is shown as follows:

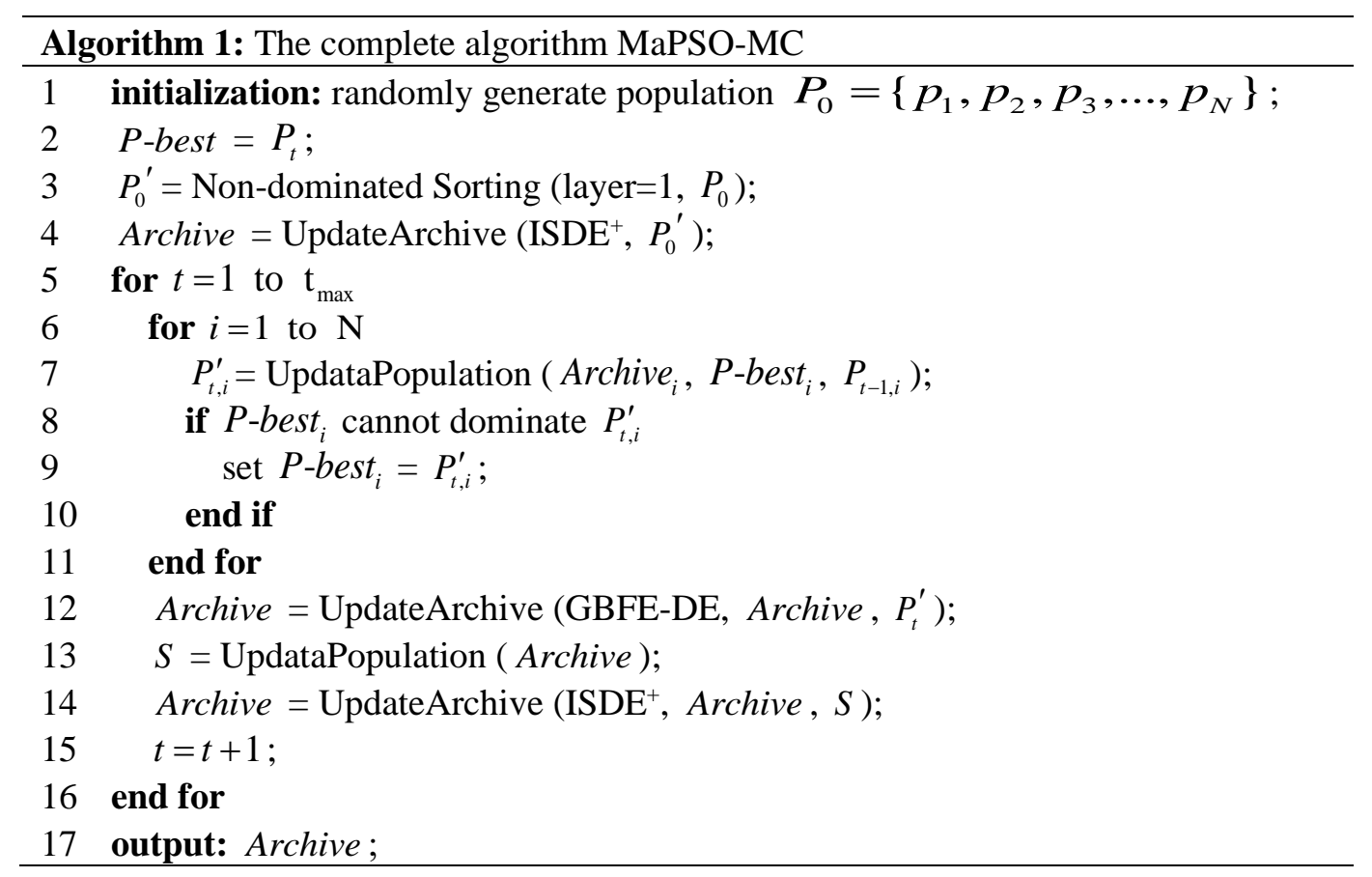

During the population update process, the individual position update will be affected by the global-best position and the personal-best position. Different from the standard PSO, a new direction is added to increase disturbance for individual updating. It is shown as (16): 


$$
v_{i}(t+1)=w v_{i}(t)+c_{1} r_{1}\left(x_{\text {pbest }}-x_{i}(t)\right)+c_{2} r_{2}\left(x_{\text {gbest }_{i}}-x_{i}(t)\right)+c_{3} r_{3}\left(x_{\text {gbest }_{i}}-x_{\text {pbest }}\right)
$$

where the meaning of the parameter is the same as (5). The archive in the pseudo code stores the global-best individuals, which is the top $10 \%$ of the individuals in the population ranked according to the evaluation criteria. The individual position update formula is shown in (6). The new term in the velocity update formula increases the direction of individual update and disturbs the individual, thereby improving the global search ability of the algorithm.

\section{MaPSO-MC for Recommendation System}

Many-objective particle swarm optimization algorithm based on multiple criteria for recommendation system (MaPSOMC-RS) is proposed for this hybrid recommendation model with constraints. At the same time, a partition-based particle swarm updating strategy (PBPS) and MaBF have been proposed to improve the search ability of the algorithm. The pseudo code of MaPSOMC-RS is shown as follows:

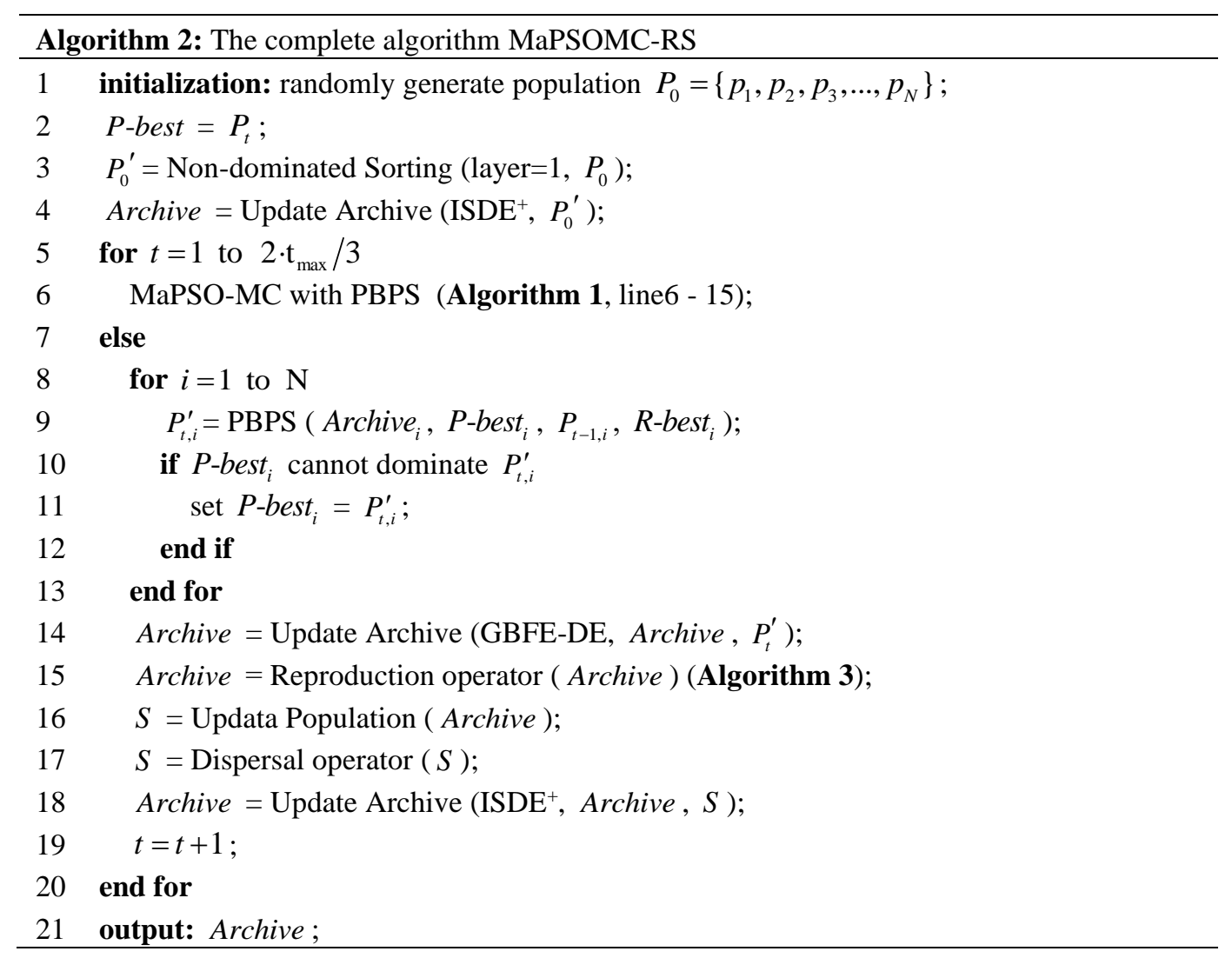

\subsection{Partition-based Particle Swarm Updating Strategy}

In the hybrid recommendation model, the combined parameter sum of the three basic recommendation algorithms is 1, and the specific constraint is shown in Formula 3. Therefore, the decision space will be divided into four regions according to the proportions of three different basic recommendation algorithms. When the proportion of a certain basic recommendation algorithm in the linear model is more than $50 \%$, the basic recommendation 
algorithm is considered as the dominant technology and is the key factor affecting the prediction rating in this individual. The entire decision space is partitioned as shown in Fig. 1:

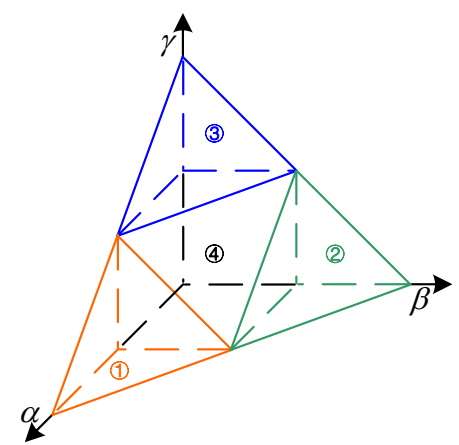

Fig. 1. Partitioned decision space

As shown in Fig. 1, regions 1-3 respectively represent the regions where user-based CF, item-based CF, and the hybrid recommendation techniques that feature combine content-based and $\mathrm{CF}$ technique. Region 4 indicates that none of the three basic recommendation techniques is dominant. It means that the three basic recommendation techniques have similar effects on model prediction ratings. The region where individual $i$ in the population is judged is as follows:

$$
R_{i, k}=\left\{\begin{array}{cc}
k=1, & \alpha_{i}>0.5 \\
k=2, & \beta_{i}>0.5 \\
k=3, & \gamma_{i}>0.5 \\
k=4, & \text { else }
\end{array} \quad \alpha_{i}+\beta_{i} \leq 1\right.
$$

After partitioning the decision space, the influence of the regional-best on their speed update will be added in the update process of the individuals in the population to improve the convergence of the algorithm. The individual speed update is shown in (18):

$$
\begin{aligned}
v_{i}(t+1)= & w v_{i}(t)+c_{1} r_{1}\left(x_{\text {pbest }_{i}}-x_{i}(t)\right)+c_{2} r_{2}\left(x_{\text {gbest }_{i}}-x_{i}(t)\right) \\
& +c_{3} r_{3}\left(x_{\text {gbest }_{i}}-x_{\text {pbest }_{i}}\right)+c_{4} r_{4}\left(x_{\text {rbest }_{i}}-x_{i}(t)\right)
\end{aligned}
$$

where $x_{r b e s t_{i}}$ represents regional-best position where individual $i$ is located. The regional-best position is the position of the best individual evaluated according to ISDE+ in this region. The influence of the regional-best position on the individual velocity update will enhance the local search ability of the algorithm.

\subsection{Many-objective Evolutionary Strategy based on Bacterial Foraging}

The MaPSO-MC simulates the foraging behavior of fish swarm and bird flock to perform global search to solve the optimal solution. However, in the process of individual update of this algorithm, the individual position update is affected by the global-best, local-best, and regional-best, and the algorithm has better global search capabilities. In the later stage of the algorithm, the individuals in the population tend to the position of the optimal solution, so an individual update strategy with stronger local search capabilities is required. 
Bacterial foraging optimization algorithm (BFO) as a swarm intelligence single-object optimization algorithm has strong local search ability. Aiming at this feature, a MaBF is constructed. In the later stage of the algorithm, individuals in the population gradually approach the optimal position. This strategy is used to perform a local search to accelerate the algorithm's convergence speed. BFO mainly includes three operations by imitating the foraging behavior of bacteria: chemotaxis operator, reproduction operator, and dispersal operator.

In the partition-based MaBF strategy, two behaviors of bacterial foraging are used: reproduction and dispersal. The chemotaxis operator (individual update strategy) in bacterial foraging is replaced by a partition-based particle swarm updating strategy. The following will specifically introduce reproduction operator and dispersal operator:

A. Reproduction Operator

During foraging, bacteria tend to gather in places where food is abundant. Bacteria in a place with a lot of food will split due to rich nutrition, while bacteria in a position lacking nutrition will die. Based on this behavior, a partition-based reproduction operator is constructed, in which the top half of the individuals in each partition are copied according to the evaluation criteria, and the latter half of the individuals are deleted.

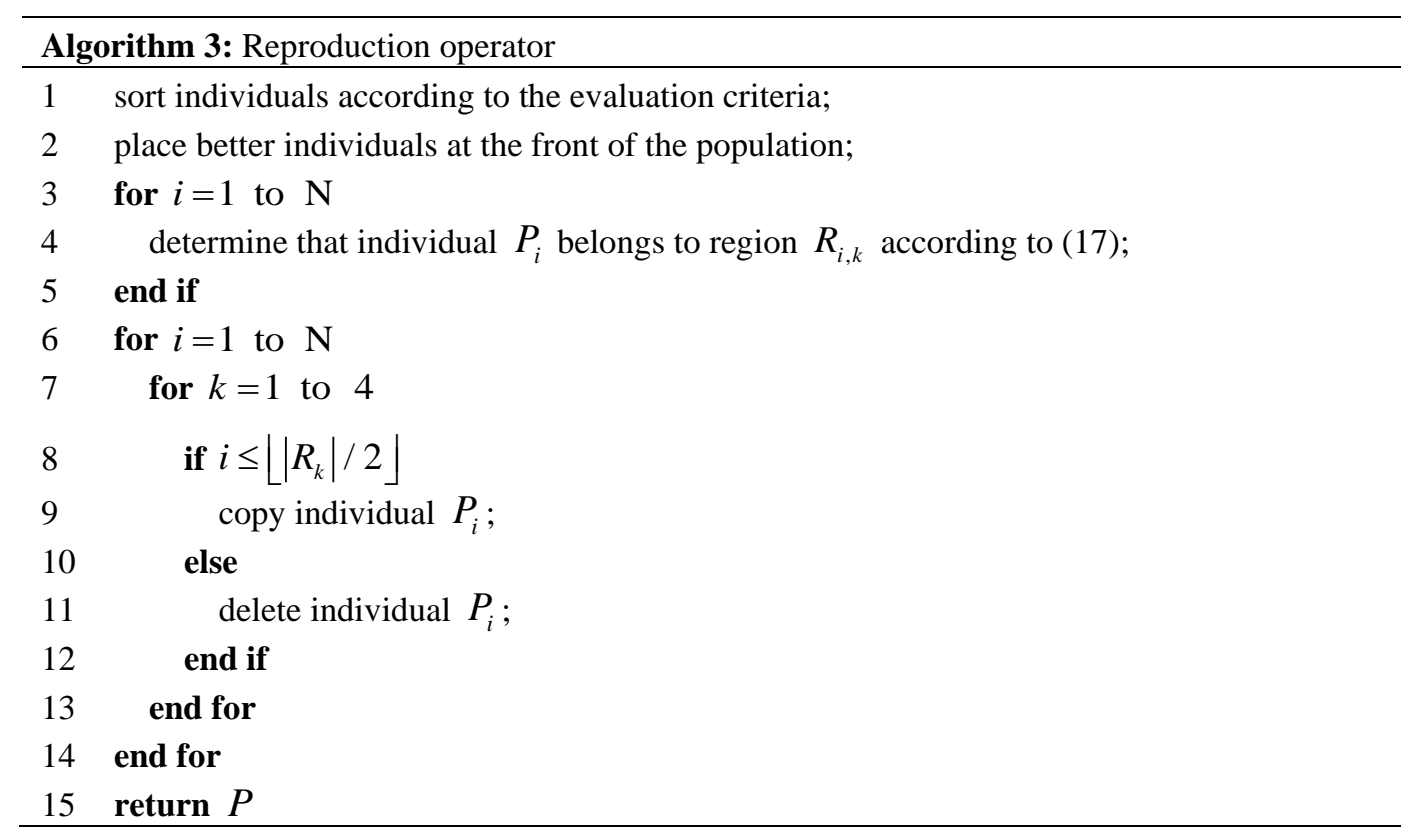

The reproduction strategy speeds up the local search ability of the algorithm by reproduction outstanding individuals in the region.

B. Dispersal Operator

Dispersal operations simulate the migration of bacterial individuals to new areas due to large food reductions or local temperature increases during bacterial foraging. In MaBF, the bacteria in the population will dispersal according to the (19):

$$
x=\left\{\begin{array}{l}
x_{\text {new }}, \text { if } q<p_{d i} \\
x, \text { otherwise }
\end{array}\right.
$$


where $p_{d i}$ is dispersal probability; $q$ is a random number between 0 and 1 subject to uniform distribution; $x_{\text {new }}$ represents the position where individual $i$ is regenerated, and the probability $P_{R_{k}}(i)$ of individual $i$ regenerating in the region $k$ is shown as follows:

$$
P_{R_{k}}(i)=\frac{\sum_{m=0}^{t} \sum_{j=0}^{N} R_{m_{-} j, k}}{N \cdot t}
$$

where $t$ and $N$ respectively represent the current generation of algorithm and population size; $R_{m_{-} j, k}$ is shown in (17). The probability of an individual regenerating in the region is based on the proportion of the number of individuals in each region of the current generation to the total number of individuals.

\section{Experiments Studies}

Firstly, MaPSO-MC is tested on the benchmark problems to verify the comprehensive performance of the algorithm. Secondly, we tested the effect of MaPSOMC-RS on the hybrid recommendation model.

\subsection{The Performance of MaPSO-MC for Benchmark Problems}

\section{A. Experimental Settings}

In this study, DTLZ1- DTLZ7 was used to test the performance of MaPSO-MC. In each problem, the algorithm is tested for performance in the range of 4 to 10 dimensions of the objective function, specifically $m \in(4,6,8,10)$. The corresponding interval of the PF of DTLZ1 is $f_{i} \in[0,0.5]$, and the corresponding interval of the PF of the remaining DTLZ problems is $[0,1]$. The dimension of the decision variable $n=m-1+k$, where $m$ is the number of objectives; $k$ is set to 5 and 20 in DTLZ1 and DTLZ7 respectively, and set to 10 in the remaining problems according to [NMPSO43].

Four MaOEAs (NSGA-III, RVEA, MaOEA-SIN, NSGAIII-GBFE) were used to compare algorithm performance. Refer to the corresponding cited reference for the parameter settings of the four comparison algorithms. In the algorithm NMPSO-MC proposed in this paper, $p_{c}$ and $p_{m}$ are set to 1 and $1 / \mathrm{n}$ as the crossover probability and mutation probability; inertia weight $w \in[0.1,0.5]$; learning parameters $c_{1}, c_{2}, c_{3} \in[1.5,2.5]$; the generation influence parameter $\theta$ in GBFE-DE is 2 .

The IGD was used as an indicator to comprehensively evaluate the convergence and diversity of the algorithm to test the performance of the algorithm. The IGD comprehensively evaluates the convergence and diversity of the algorithm by calculating the average distance from the individual on the PF to the closest individual in the algorithm solution set. A smaller IGD represents a better performance of the algorithm.

B. The Performance of MaPSO-MC in Benchmark Problems

The five algorithms in the comparison experiment were independently run 30 times, and the 
results are shown in Table $\mathbf{1 .}$

Table 1. The Comparisons results of MaPSO-MC with four current MaOEAs

\begin{tabular}{|c|c|c|c|c|c|c|}
\hline Problem & M & NSGA-III & RVEA & MaOEA-SIN & NSGAIII-GBFE & NMPSO-MC \\
\hline \multirow{4}{*}{ DTLZ1 } & 4 & $2.3738 \mathrm{e}+1(5.45 \mathrm{e}+0)+$ & $1.7928 \mathrm{e}+1(6.25 \mathrm{e}+0)+$ & $1.5735 \mathrm{e}+1(4.19 \mathrm{e}+0)+$ & $1.8255 \mathrm{e}+1(3.97 \mathrm{e}+0)+$ & $2.6238 \mathrm{e}+1(5.03 \mathrm{e}+0)$ \\
\hline & 6 & $2.1117 \mathrm{e}+1(6.76 \mathrm{e}+0)+$ & $1.6878 \mathrm{e}+1(6.13 \mathrm{e}+0)+$ & $1.4082 e+1(3.37 e+0)+$ & $1.7678 \mathrm{e}+1(4.70 \mathrm{e}+0)+$ & $2.5729 \mathrm{e}+1(5.16 \mathrm{e}+0)$ \\
\hline & 8 & $2.7190 \mathrm{e}+1(7.75 \mathrm{e}+0)=$ & $1.6227 e+1(3.91 e+0)+$ & $1.7509 \mathrm{e}+1(3.98 \mathrm{e}+0)+$ & $1.8989 \mathrm{e}+1(5.26 \mathrm{e}+0)+$ & $2.3584 \mathrm{e}+1(4.66 \mathrm{e}+0)$ \\
\hline & 10 & $2.6669 \mathrm{e}+1(6.40 \mathrm{e}+0)=$ & $2.3039 \mathrm{e}+1(8.29 \mathrm{e}+0)=$ & $2.1247 \mathrm{e}+1(5.43 \mathrm{e}+0)=$ & $2.3286 \mathrm{e}+1(7.50 \mathrm{e}+0)=$ & $2.3310 \mathrm{e}+1(5.03 \mathrm{e}+0)$ \\
\hline \multirow{4}{*}{ DTLZ2 } & 4 & $4.9890 \mathrm{e}-1(4.24 \mathrm{e}-2)-$ & $5.2454 \mathrm{e}-1(3.22 \mathrm{e}-2)-$ & $4.6360 \mathrm{e}-1(2.49 \mathrm{e}-2)=$ & $4.8997 \mathrm{e}-1(4.23 \mathrm{e}-2)-$ & $4.5841 \mathrm{e}-1(3.54 \mathrm{e}-2)$ \\
\hline & 6 & $7.3121 \mathrm{e}-1(3.49 \mathrm{e}-2)-$ & $7.6044 \mathrm{e}-1(4.23 \mathrm{e}-2)-$ & $7.0021 \mathrm{e}-1(3.37 \mathrm{e}-2)-$ & $7.2872 \mathrm{e}-1(3.79 \mathrm{e}-2)-$ & $6.3120 \mathrm{e}-1(3.09 \mathrm{e}-2)$ \\
\hline & 8 & $8.9765 \mathrm{e}-1(5.10 \mathrm{e}-2)-$ & $9.5335 \mathrm{e}-1(4.86 \mathrm{e}-2)-$ & $8.6296 \mathrm{e}-1(3.24 \mathrm{e}-2)-$ & $9.1071 \mathrm{e}-1(4.58 \mathrm{e}-2)-$ & $8.0955 e-1(4.72 e-2)$ \\
\hline & 10 & $9.9525 \mathrm{e}-1(4.63 \mathrm{e}-2)=$ & $9.9484 \mathrm{e}-1(3.26 \mathrm{e}-2)=$ & $9.9641 \mathrm{e}-1(3.44 \mathrm{e}-2)=$ & $9.9169 \mathrm{e}-1(3.08 \mathrm{e}-2)=$ & $9.9980 \mathrm{e}-1(3.95 \mathrm{e}-2)$ \\
\hline \multirow{4}{*}{ DTLZ3 } & 4 & $2.5517 \mathrm{e}+2(4.99 \mathrm{e}+1)-$ & $2.2925 \mathrm{e}+2(5.13 \mathrm{e}+1)-$ & $1.9261 \mathrm{e}+2(3.33 \mathrm{e}+1)=$ & $2.2303 \mathrm{e}+2(3.90 \mathrm{e}+1)-$ & $2.0093 \mathrm{e}+2(2.13 \mathrm{e}+1)$ \\
\hline & 6 & $3.1101 \mathrm{e}+2(5.07 \mathrm{e}+1)-$ & $2.3659 \mathrm{e}+2(4.56 \mathrm{e}+1)-$ & $1.8485 \mathrm{e}+2(4.00 \mathrm{e}+1)+$ & $2.3485 \mathrm{e}+2(4.84 \mathrm{e}+1)-$ & $2.0720 \mathrm{e}+2(1.27 \mathrm{e}+1)$ \\
\hline & 8 & $3.4705 \mathrm{e}+2(6.20 \mathrm{e}+1)-$ & $2.2936 \mathrm{e}+2(4.63 \mathrm{e}+1)-$ & $2.2601 \mathrm{e}+2(4.38 \mathrm{e}+1)-$ & $2.3035 \mathrm{e}+2(5.81 \mathrm{e}+1)-$ & $2.0250 \mathrm{e}+2(1.28 \mathrm{e}+1)$ \\
\hline & 10 & $3.6539 \mathrm{e}+2(5.70 \mathrm{e}+1)-$ & $2.9671 \mathrm{e}+2(5.80 \mathrm{e}+1)-$ & $2.8636 \mathrm{e}+2(5.36 \mathrm{e}+1)-$ & $2.9767 \mathrm{e}+2(6.27 \mathrm{e}+1)-$ & $2.1443 \mathrm{e}+2(1.02 \mathrm{e}+1)$ \\
\hline \multirow{4}{*}{ DTLZ4 } & 4 & $8.7076 \mathrm{e}-1$ (8.71e-2) - & $7.3519 \mathrm{e}-1(6.32 \mathrm{e}-2)+$ & $8.4724 \mathrm{e}-1(1.05 \mathrm{e}-1)=$ & $9.3023 \mathrm{e}-1(1.06 \mathrm{e}-1)-$ & $8.1556 \mathrm{e}-1(8.79 \mathrm{e}-2)$ \\
\hline & 6 & $1.0529 \mathrm{e}+0(1.14 \mathrm{e}-1)-$ & $1.0818 \mathrm{e}+0(9.30 \mathrm{e}-2)-$ & $1.0532 \mathrm{e}+0(7.14 \mathrm{e}-2)-$ & $1.0634 \mathrm{e}+0(7.89 \mathrm{e}-2)-$ & $9.7393 \mathrm{e}-1(6.96 \mathrm{e}-2)$ \\
\hline & 8 & $1.1001 \mathrm{e}+0(5.89 \mathrm{e}-2)-$ & $1.1436 \mathrm{e}+0(8.29 \mathrm{e}-2)-$ & $1.0709 \mathrm{e}+0(5.05 \mathrm{e}-2)=$ & $1.1013 \mathrm{e}+0(7.11 \mathrm{e}-2)-$ & $1.0662 e+0(6.74 e-2)$ \\
\hline & 10 & $1.1453 \mathrm{e}+0(3.94 \mathrm{e}-2)=$ & $1.1575 \mathrm{e}+0(5.80 \mathrm{e}-2)=$ & $1.1483 \mathrm{e}+0(5.26 \mathrm{e}-2)=$ & $1.1466 \mathrm{e}+0(4.10 \mathrm{e}-2)=$ & $1.1489 \mathrm{e}+0(4.84 \mathrm{e}-2)$ \\
\hline \multirow{4}{*}{ DTLZ5 } & 4 & $3.4183 \mathrm{e}-1(3.74 \mathrm{e}-2)-$ & $4.2028 \mathrm{e}-1(7.17 \mathrm{e}-2)-$ & $2.9460 \mathrm{e}-1(2.94 \mathrm{e}-2)-$ & $3.2701 \mathrm{e}-1(5.37 \mathrm{e}-2)-$ & $2.7665 \mathrm{e}-1(4.19 \mathrm{e}-2)$ \\
\hline & 6 & $3.8591 \mathrm{e}-1(4.29 \mathrm{e}-2)-$ & $4.8829 \mathrm{e}-1(6.05 \mathrm{e}-2)-$ & $3.5653 \mathrm{e}-1(4.65 \mathrm{e}-2)-$ & $3.9427 \mathrm{e}-1(4.41 \mathrm{e}-2)-$ & $2.9250 \mathrm{e}-1(3.70 \mathrm{e}-2)$ \\
\hline & 8 & $3.7108 \mathrm{e}-1$ (4.96e-2) - & $5.5594 \mathrm{e}-1(8.92 \mathrm{e}-2)-$ & $3.4830 \mathrm{e}-1(3.44 \mathrm{e}-2)-$ & $3.7488 \mathrm{e}-1(4.76 \mathrm{e}-2)-$ & $2.8392 \mathrm{e}-1(3.62 \mathrm{e}-2)$ \\
\hline & 10 & $3.7670 \mathrm{e}-1(3.94 \mathrm{e}-2)=$ & $3.7352 \mathrm{e}-1(3.29 \mathrm{e}-2)=$ & $3.6701 \mathrm{e}-1(5.21 \mathrm{e}-2)=$ & $3.5780 \mathrm{e}-1(3.51 \mathrm{e}-2)=$ & $3.6665 \mathrm{e}-1(3.05 \mathrm{e}-2)$ \\
\hline \multirow{4}{*}{ DTLZ6 } & 4 & $7.9897 \mathrm{e}+0(3.16 \mathrm{e}-1)-$ & $8.0620 \mathrm{e}+0(2.04 \mathrm{e}-1)-$ & $7.4449 \mathrm{e}+0(4.72 \mathrm{e}-1)-$ & $7.9359 \mathrm{e}+0(2.98 \mathrm{e}-1)-$ & $2.9607 e+0(6.39 e-1)$ \\
\hline & 6 & $8.2003 \mathrm{e}+0(2.77 \mathrm{e}-1)-$ & $8.4863 \mathrm{e}+0(2.25 \mathrm{e}-1)-$ & $7.9037 \mathrm{e}+0(1.86 \mathrm{e}-1)-$ & $8.1085 \mathrm{e}+0(2.63 \mathrm{e}-1)-$ & $3.2891 \mathrm{e}+0(7.28 \mathrm{e}-1)$ \\
\hline & 8 & $8.1921 \mathrm{e}+0(2.30 \mathrm{e}-1)-$ & $8.5131 \mathrm{e}+0(2.53 \mathrm{e}-1)-$ & $7.8934 \mathrm{e}+0(3.62 \mathrm{e}-1)-$ & $8.1122 \mathrm{e}+0(2.03 \mathrm{e}-1)-$ & $3.4864 e+0(5.86 e-1)$ \\
\hline & 10 & $8.4063 e+0(1.08 \mathrm{e}-1)-$ & $8.3784 e+0(1.07 e-1)-$ & $8.3787 \mathrm{e}+0(1.02 \mathrm{e}-1)=$ & $8.3893 e+0(1.17 e-1)-$ & $8.3075 e+0(1.21 e-1)$ \\
\hline \multirow{4}{*}{ DTLZ7 } & 4 & $1.0717 \mathrm{e}+1(1.05 \mathrm{e}+0)-$ & $9.6135 \mathrm{e}+0(9.21 \mathrm{e}-1)-$ & $9.6701 e+0(7.35 e-1)-$ & $1.1336 \mathrm{e}+1(8.93 \mathrm{e}-1)-$ & $7.2254 \mathrm{e}+0(2.37 \mathrm{e}+0)$ \\
\hline & 6 & $1.7051 \mathrm{e}+1(1.58 \mathrm{e}+0)-$ & $1.6741 \mathrm{e}+1(1.82 \mathrm{e}+0)-$ & $1.6907 \mathrm{e}+1(1.26 \mathrm{e}+0)-$ & $1.7475 \mathrm{e}+1(1.39 \mathrm{e}+0)-$ & $1.2188 \mathrm{e}+1(2.04 \mathrm{e}+0)$ \\
\hline & 8 & $2.3103 \mathrm{e}+1(1.63 \mathrm{e}+0)-$ & $2.3423 \mathrm{e}+1(1.93 \mathrm{e}+0)-$ & $2.2044 \mathrm{e}+1(2.12 \mathrm{e}+0)-$ & $2.4962 \mathrm{e}+1(1.43 \mathrm{e}+0)-$ & $1.9318 \mathrm{e}+1(2.79 \mathrm{e}+0)$ \\
\hline & 10 & $2.9963 \mathrm{e}+1(2.04 \mathrm{e}+0)-$ & $2.8877 \mathrm{e}+1(2.96 \mathrm{e}+0)=$ & $2.8165 \mathrm{e}+1(2.38 \mathrm{e}+0)=$ & $2.8476 \mathrm{e}+1(2.30 \mathrm{e}+0)=$ & $2.8460 \mathrm{e}+1(2.71 \mathrm{e}+0)$ \\
\hline \multicolumn{2}{|c|}{$+/-1=$} & $2 / 21 / 5$ & $4 / 19 / 5$ & $4 / 14 / 10$ & $3 / 20 / 5$ & \\
\hline
\end{tabular}

The bold results in Table 1 represent that the algorithm has the best performance in a certain dimension of a certain problem set. "+", “-", and "=" represent, respectively, the number of comparison algorithms that are better, worse, and similar than the results of the algorithm proposed in the paper. It can be clearly seen from Table 1 that MaPSO-MC has obvious advantages in solving the various dimensions of the DTLZ2 - DTLZ7 problems. Since DTLZ2-DTLZ5 and DTLZ7 focus on the ability of algorithms to deal with different shapes and distribution problems, the algorithms have excellent performance in this regard. DTLZ1 and DTLZ6 are used to evaluate the convergence ability of the algorithm. Although the proposed algorithm has general results on DTLZ1, it also has a good performance in dealing 
with the problems with many-objectives. DTLZ1 mainly focuses on evaluating the ability of algorithms to optimize multi-modal and linear problems. But this linear relationship refers to the relationship between the objectives, and the linear relationship in the model is a way of weighting three basic recommendation techniques. Therefore, although this algorithm performs slightly worse on DTLZ1, it does not prove that the performance of the optimized model is also poor. At the same time, MaOEA-SIN and NSGAIII-GBFE are newly proposed algorithms in recent years. Even though some of the results are better than MaPSO-MC, most of those differences are small.

\subsection{The Performance of MaPSOMC-RS for Hybrid Recommendation model}

\section{A. Experimental Settings}

As the most classic data set for testing recommendation performance, MovieLens is used to evaluate the effectiveness of the hybrid recommendation model. "MovieLens 1M Dataset" with 1,000,209 ratings from 6040 users on 3952 movies is adopted to test performance (it can be downloaded from https://grouplens.org/datasets/movielens/). This data set contains 5 ratings. In this experiment, we consider that items with a predicted rating greater than 3 are the items that users prefer. Different test set and training set division methods are used to obtain MovieLens1, MovieLens2, MovieLens3, and MovieLens4.

The precision and recall are both indicators used to evaluate the accuracy of recommendations, and to a certain extent these two indicators will conflict, so the F-measure is used to evaluate the accuracy of recommendations. It can be calculated as follow:

$$
F-\text { measure }=\frac{2 \cdot \text { recall } \cdot \text { precision }}{\text { recall }+ \text { precision }}
$$

The F-measure, diversity, and novelty of the recommendation system is the average value of the F-measure, diversity, and novelty of each target user recommendation list in the system. As an indicator for evaluating the entire system, the coverage is calculated directly after recommendation for all users in the system.

Moreover, the recommended module uses Python to run on Pycharm, the optimization algorithm module uses Matlab to run on MATLAB, and the interface to make the two platforms interoperate. In the algorithm parameter setting, the decision variable $D=2$; population size $m=35$; the maximum generation $t_{\max }=20$; items that have been rated more than 500 times are defined as popular items; the remaining parameter settings of the algorithm are the same as in section 5.1.

\section{$B$. the performance of MaPSOMC-RS in hybrid recommendation model}

Table 2 shows the comparison effect of the NSGA-III [24], RVEA [25], MaOEA-SIN [38], NSGAIII-GBFE [34], and MaPSOMC-RS on the F-measure, diversity, novelty, and coverage of the recommendation model. At the same time, since each algorithm will produce a set of solutions (providing users with multiple recommendation lists), each recommendation list has different performance in the 4 evaluation indicators. Therefore, the maximum, minimum and average of the solution set on each evaluation indicator are adopted to evaluate the performance of different algorithms to solve the model. 
Table 2. Performance of five algorithms in this model

\begin{tabular}{|c|c|c|c|c|c|}
\hline \multicolumn{2}{|c|}{ Algorithm } & \multirow{2}{*}{$\begin{array}{c}\text { F-measure } \\
95.996 \%\end{array}$} & \multirow{2}{*}{$\begin{array}{c}\text { Diversity } \\
0.90439\end{array}$} & \multirow{2}{*}{$\begin{array}{l}\text { Novelty } \\
37.805 \%\end{array}$} & \multirow{2}{*}{$\begin{array}{r}\text { Coverage } \\
77.249 \%\end{array}$} \\
\hline & User-based CF & & & & \\
\hline recommendation & Item-based CF & $96.576 \%$ & 0.90772 & $40.187 \%$ & $87.019 \%$ \\
\hline & Content-based & $96.875 \%$ & 0.90548 & $38.861 \%$ & $83.595 \%$ \\
\hline \multirow{5}{*}{ Best } & MaPSOMC-RS & $97.376 \%$ & 0.90981 & $41.843 \%$ & $\mathbf{8 8 . 7 8 7 \%}$ \\
\hline & NSGAIII-GBFE-RS & $97.336 \%$ & 0.90980 & $41.457 \%$ & $87.515 \%$ \\
\hline & NSGA-III & $97.190 \%$ & 0.90618 & $41.700 \%$ & $87.699 \%$ \\
\hline & RVEA & $97.300 \%$ & 0.90708 & $41.317 \%$ & $87.539 \%$ \\
\hline & MaOEA-SIN & $97.340 \%$ & 0.90958 & $42.040 \%$ & $88.515 \%$ \\
\hline \multirow{5}{*}{ Worst } & MaPSOMC-RS & $96.473 \%$ & 0.90873 & $39.372 \%$ & $\mathbf{8 1 . 8 3 1 \%}$ \\
\hline & NSGAIII-GBFE-RS & $96.652 \%$ & 0.90866 & $39.274 \%$ & $81.464 \%$ \\
\hline & NSGA-III & $96.422 \%$ & 0.90450 & $39.022 \%$ & $80.104 \%$ \\
\hline & RVEA & $96.532 \%$ & 0.90543 & $39.212 \%$ & $80.088 \%$ \\
\hline & MaOEA-SIN & $96.572 \%$ & 0.90772 & $39.362 \%$ & $80.168 \%$ \\
\hline \multirow{5}{*}{ Mean } & MaPSOMC-RS & $97.063 \%$ & 0.90935 & $40.769 \%$ & $85.905 \%$ \\
\hline & NSGAIII-GBFE-RS & $97.043 \%$ & 0.90930 & $40.644 \%$ & $85.542 \%$ \\
\hline & NSGA-III & $96.873 \%$ & 0.90560 & $40.315 \%$ & $84.915 \%$ \\
\hline & RVEA & $96.985 \%$ & 0.90648 & $40.359 \%$ & $84.368 \%$ \\
\hline & MaOEA-SIN & $97.023 \%$ & 0.90897 & $40.655 \%$ & $84.989 \%$ \\
\hline
\end{tabular}

From Table 2, it can be shown that MaPSOMC-RS has better overall performance when solving hybrid recommendation models. The average performance of the solutions obtained by MaPSOMC-RS in solving the model is superior to that of the comparison algorithm in the four evaluation indicators. Compared with the other four algorithms, the maximum and minimum solutions obtained by the proposed algorithm also have better results. Although MaPSOMC-RS does not achieve optimal results on some evaluation indicators, it is not much different from the optimal results. The boxplots of the five algorithms on different evaluation indicators are shown in Fig. 2: 


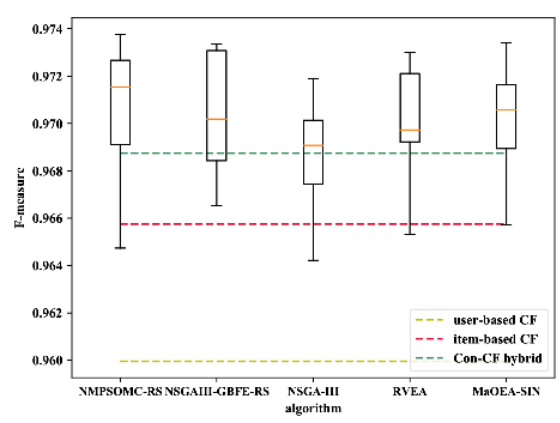

(a)

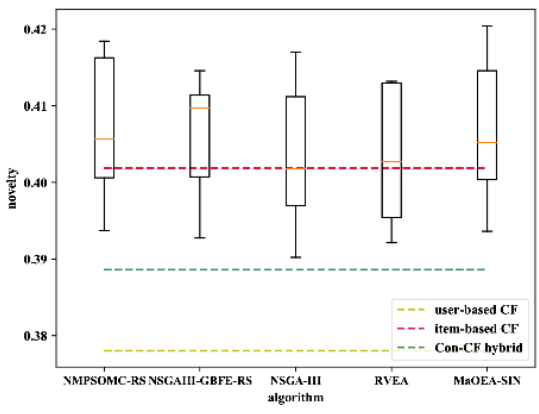

(c)

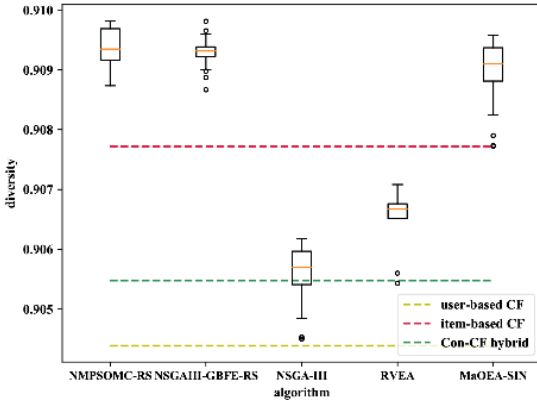

(b)

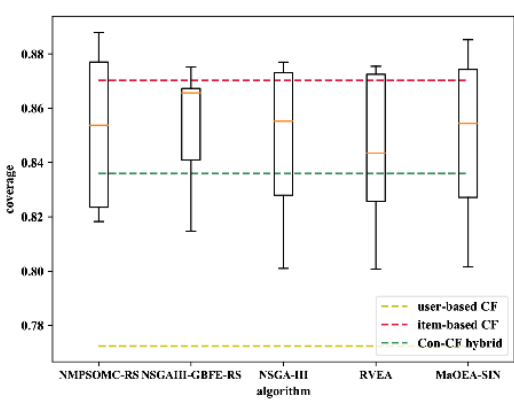

(d)

Fig. 2. The boxplots of the five algorithms on: (a) F-measure, (b) diversity, (c) novelty, (d) coverage

In Fig. 2, there are four black lines and one red line in each box respectively representing the maximum value, the first quartile value, the last quartile value, the minimum value and the median value of the data in this box, and the circle in the figure represents the discrete individual. The three dotted lines represent the performance of the three basic recommended technologies on different evaluation indicators. From the perspective of median value and convergence, compared to the other four algorithms, MaPSOMC-RS has the best effect. Since MaPSO contains the least discrete individuals, the algorithm has the strongest stability. By using a variety of evaluation criteria, MaPSOMC-RS can focus on the convergence of the algorithm in the early stage of the algorithm, focus on the diversity in the later stage of the algorithm, and comprehensively evaluating the multiple performance of the algorithm. The regional-best also has an impact on individual update. Therefore, MaPSOMC-RS has excellent convergence and recommendation results when dealing with this problem.

The number of users in this data set is greater than the number of items, and Fig. 2 also proves that item-based $\mathrm{CF}$ can provide better recommendation lists on multiple evaluation indicators. Meanwhile, each solution in the population represents a recommendation list, and the decision maker can select the required training parameters according to his own preferences to make recommendations for the user.

\subsection{The Performance of the PBPS and MaBF}

Table 3 shows performance comparison MaPSOMC-RS (with PBPS and MaBF) and MaPSO$\mathrm{MC}$ (without PBPS and MaBF) in five evaluation indicators. 
Table 3. The performance of PBPS and MaBF

\begin{tabular}{|c|c|c|c|c|c|c|c|}
\hline \multirow{3}{*}{ Test set } & \multirow{2}{*}{ Evaluation indicators } & \multicolumn{3}{|c|}{ MaPSOMC-RS } & \multicolumn{3}{c|}{ MaPSO-MC } \\
\cline { 2 - 7 } & & Best & Mean & Worst & Best & Mean & Worst \\
\hline \multirow{3}{*}{ MovieLens2 } & F-measure & $95.238 \%$ & $\mathbf{9 5 . 1 0 1 \%}$ & $94.698 \%$ & $95.224 \%$ & $95.096 \%$ & $94.713 \%$ \\
\cline { 2 - 8 } & Diversity & 0.93112 & $\mathbf{0 . 9 2 9 8 4}$ & 0.92876 & 0.93082 & 0.92973 & 0.92859 \\
\cline { 2 - 8 } & Novelty & $42.187 \%$ & $\mathbf{4 1 . 5 8 3 \%}$ & $39.713 \%$ & $41.825 \%$ & $41.430 \%$ & $39.238 \%$ \\
\hline \multirow{3}{*}{ MovieLens3 } & Coverage & $90.021 \%$ & $\mathbf{8 8 . 2 8 7 \%}$ & $82.565 \%$ & $89.398 \%$ & $87.901 \%$ & $82.368 \%$ \\
\cline { 2 - 8 } & F-measure & $97.370 \%$ & $\mathbf{9 7 . 1 4 7 \%}$ & $96.640 \%$ & $97.239 \%$ & $97.001 \%$ & $96.631 \%$ \\
\cline { 2 - 8 } & Diversity & $93.887 \%$ & $\mathbf{0 . 9 3 7 2 3}$ & 0.93611 & 0.93691 & 0.93547 & 0.93448 \\
\cline { 2 - 8 } & Novelty & $44.241 \%$ & $\mathbf{4 3 . 6 2 0 \%}$ & $41.122 \%$ & $43.983 \%$ & $43.087 \%$ & $40.981 \%$ \\
\hline \multirow{3}{*}{ Coverage } & $89.798 \%$ & $89.467 \%$ & $84.261 \%$ & $89.802 \%$ & $\mathbf{8 8 . 5 4 9 \%}$ & $84.137 \%$ \\
\cline { 2 - 7 } & F-measure & $96.365 \%$ & $\mathbf{9 5 . 9 8 5 \%}$ & $95.450 \%$ & $96.128 \%$ & $95.776 \%$ & $95.300 \%$ \\
\cline { 2 - 7 } & Diversity & 0.93766 & $\mathbf{0 . 9 3 7 0 1}$ & 0.93571 & 0.93706 & 0.93677 & 0.93493 \\
\cline { 2 - 7 } & Novelty & $43.022 \%$ & $\mathbf{4 2 . 6 4 2 \%}$ & $40.831 \%$ & $42.967 \%$ & $42.538 \%$ & $41.149 \%$ \\
\cline { 2 - 7 } & Coverage & $87.421 \%$ & $\mathbf{8 6 . 2 4 5 \%}$ & $81.877 \%$ & $87.115 \%$ & $86.015 \%$ & $81.059 \%$ \\
\hline
\end{tabular}

It has been proven that the PBPS and MaBF are effective for this hybrid recommendation model. In different data set, the solution set optimized by MaPSOMC-RS is almost better than MaPSO-MC in the best, mean and worst results. PBPS considers the influence of region-best during the individual update process, and MaBF accelerates the local search speed of the algorithm in the later stage of the algorithm.

The experimental results show that PBPS and MaBF improve the overall recommended performance. These strategies provide users with accurate, diverse and novel recommendations by accelerating the search speed of the algorithm, and at the same time can help suppliers recommend more products to increase profits.

\section{Conclusion}

In this paper, in order to solve the hybrid recommendation model with constraints, MaPSO$\mathrm{MC}$, as a MaOEA with swarm intelligence optimization, includes two evaluation criteria is proposed to improve the recommendation performance of hybrid models. Two evaluation criteria, ISDE ${ }^{+}$and GBFE-DE, are coupled and used to comprehensively evaluate individual performance. Experimental results also prove that this algorithm has good performance in solving the benchmark problems and the recommendation problem. At the same time, in view of the feature that this decision space can be partitioned, the PBPS and MaBF is designed to improve the local search speed in the later stage of the algorithm. MaPSOMC-RS, which is modified to address the characteristics of the model, shows significant advantages in addressing this recommended model. Therefore, this algorithm can provide users with accurate, diverse, novel, and as many types of items as possible when solving the hybrid recommendation model. 


\section{Acknowledgement}

This work was supported by National Natural Science Foundation of China (Grant No. 61806138), Key R\&D program of Shanxi Province (High Technology, Grant No. 201903D121119), and Postgraduate Education Innovation Project of Shanxi Province under Grant (Grant No.2020SY437).

\section{References}

[1] J. Bobadilla, F. Ortega, A. Hernando, and A. Gutiérrez, "Recommender systems survey," Knowledge-Based Systems, vol. 46, pp. 109-132, 2013. Article (CrossRef Link)

[2] P. Winoto and T. Y. Tang, "The role of user mood in movie recommendations," Expert Systems with Applications, vol. 37, no. 8, pp. 6086-6092, 2010. Article (CrossRef Link)

[3] S. K. Lee, Y. H. Cho, and S. H. Kim, "Collaborative filtering with ordinal scale-based implicit ratings for mobile music recommendations," Information Sciences, vol. 180, no. 11, pp. 2142-2155, 2010. Article (CrossRef Link)

[4] K. F. Yeung and Y. Yang, "A Proactive Personalized Mobile News Recommendation System," Developments in E-systems Engineering, pp. 207-212, 2010. Article (CrossRef Link)

[5] H. Azari and M. Moradipour, "Using kernel-based collocation methods to solve a delay partial differential equation with application to finance," International journal of computing science and mathematics, vol. 10, no. 1, pp. 105-114, 2019. Article (CrossRef Link)

[6] S. T. Cheng, G. J. Horng, and C. L. Chou, "The Adaptive Recommendation Mechanism for Distributed Group in Mobile Environments," IEEE Transactions on Systems Man and Cybernetics Part C, vol. 42, no. 6, pp. 1081-1092, 2012. Article (CrossRef Link)

[7] P. B. Throat, R. M. Goudar, and S. Barve, "Survey on Collaborative Filtering, Content-based Filtering and Hybrid Recommendation System," International Journal of Computer Applications, vol. 110, no. 4, pp. 31-36, 2015. Article (CrossRef Link)

[8] A. B. Barragáns-Martínez, E. Costa-Montenegro, J. C. Burguillo, M. Rey-López, F. A. MikicFonte, and A. Peleteiro, "A hybrid content-based and item-based collaborative filtering approach to recommend TV programs enhanced with singular value decomposition," Information Sciences, vol. 180, no. 22, pp. 4290-4311, 2010. Article (CrossRef Link)

[9] C. Xu, A. S. Ding, and S. S. Liao, "A privacy-preserving recommendation method based on multiobjective optimisation for mobile users," International Journal of Bio-Inspired Computation, vol. 16, no. 1, pp. 23-32, 2020. Article (CrossRef Link)

[10] N. Hurley and M. Zhang, "Novelty and Diversity in Top-N Recommendation--Analysis and Evaluation," ACM Transactions on Internet Technology, vol. 10, no. 4, 2011. Article (CrossRef Link)

[11] B. Geng, L. Li, L. Jiao, M. Gong, Q. Cai, and Y. Wu, "NNIA-RS: A multi-objective optimization based recommender system," Physica A Statistical Mechanics and Its Applications, vol. 424, pp. 383-397, 2015. Article (CrossRef Link)

[12] B. M. Sarwar, G. Karypis, J. A. Konstan, and J. Riedl, "Item-based collaborative filtering recommendation algorithms," in Proc. of $10^{\text {th }}$ International Conference on World Wide Web, pp. 285-295, 2001. Article (CrossRef Link)

[13] Y. Zuo, M. Gong, J. Zeng, L. Ma, and L. Jiao, "Personalized Recommendation Based on Evolutionary Multi-Objective Optimization [Research Frontier]," vol. 10, no. 1, pp. 52-62, 2015. Article (CrossRef Link)

[14] Z. Cui, X. Xu, F. Xue, X. Cai, Y. Cao, W. Zhang, and J. Chen, "Personalized Recommendation System Based on Collaborative Filtering for IoT Scenarios," IEEE Transactions on Services Computing, vol. 13, no. 4, pp. 685-695, 2020. Article (CrossRef Link)

[15] X. Cai, Z. Hu, P. Zhao, W. Zhang, and J. Chen, "A hybrid recommendation system with manyobjective evolutionary algorithm," Expert Systems with Applications, vol. 159, 2020. Article (CrossRef Link) 
[16] M. S. Mohamed and H. Duan, "Flight control system design using adaptive pigeon-inspired optimisation," International Journal of Bio-Inspired Computation, vol. 16, no. 3, pp. 133-147, 2020. Article (CrossRef Link)

[17] W. Deng, J. Xu, Y. Song, and H. Zhao, "An effective improved co-evolution ant colony optimisation algorithm with multi-strategies and its application," International Journal of BioInspired Computation, vol. 16, no. 3, pp. 158-170, 2020. Article (CrossRef Link)

[18] Y. Zhang, X. Cai, H. Zhu, and Y. Xu, "Application an improved swarming optimisation in attribute reduction," International Journal of Bio-Inspired Computation, vol. 16, no. 4, pp. 213-219, 2020. Article (CrossRef Link)

[19] L. Zhang, J. Xia, F. Cheng, J. Qiu, and X. Zhang, "Multi-Objective Optimization of Critical Node Detection Based on Cascade Model in Complex Networks," IEEE Transactions on Network Science and Engineering, vol. 7, no. 3, pp. 2052-2066, 2020. Article (CrossRef Link)

[20] Z. Cui, Y. Zhao, Y. Cao, X. Cai, W. Zhang, and J. Chen, "Malicious code detection under 5G HetNets based on multi-objective RBM model," IEEE Network, 2020, Article (CrossRef Link)

[21] Z. Cui, F. Xue, S. Zhang, X. Cai, Y. Cao, W. Zhang, and J. Chen, "A Hybrid BlockChain-Based Identity Authentication Scheme for Multi-WSN," IEEE Transactions on Services Computing, vol. 13, no.2, pp. 241-251, 2020. Article (CrossRef Link)

[22] Z. Cui, J. Zhang, D. Wu, X. Cai, H. Wang, W. Zhang, and J. Chen, "Hybrid many-objective particle swarm optimization algorithm for green coal production problem," Information Sciences, vol. 518, pp. 256-271, 2020. Article (CrossRef Link)

[23] X. Cai, S. Geng, D. Wu, J. Cai, and J. Chen, "A Multi-cloud Model based Many-objective Intelligent Algorithm for Efficient Task Scheduling in Internet of Things," IEEE Internet of Things Journal, 2020. Article (CrossRef Link)

[24] K. Deb and H. Jain, "An Evolutionary Many-Objective Optimization Algorithm Using ReferencePoint-Based Nondominated Sorting Approach, Part I: Solving Problems With Box Constraints," IEEE Transactions on Evolutionary Computation, vol. 18, no. 4, pp. 577-601, 2014. Article (CrossRef Link)

[25] R. Cheng, Y. Jin, M. Olhofer, and B. Sendhoff, "A Reference Vector Guided Evolutionary Algorithm for Many-Objective Optimization," IEEE Transactions on Evolutionary Computation, vol. 20, no. 5, pp. 773-791, 2016. Article (CrossRef Link)

[26] S. Yang, M. Li, X. Liu, and J. Zheng, "A Grid-Based Evolutionary Algorithm for Many-Objective Optimization," IEEE Transactions on Evolutionary Computation, vol. 17, no. 5, pp. 721-736, 2013. Article (CrossRef Link)

[27] Q. Lin, S. Liu, Q. Zhu, C. Tang, R. Song, J. Chen, C. A. Coello, K. C. Wong, and J. Zhang, "Particle Swarm Optimization With a Balanceable Fitness Estimation for Many-Objective Optimization Problems," IEEE Transactions on Evolutionary Computation, vol. 22, no. 1, pp. 32-46, 2016. Article (CrossRef Link)

[28] A. Prajapati and S. Kumar, "PSO-MoSR: a PSO-based multi-objective software remodularisation," International Journal of Bio-Inspired Computation, vol. 15, no. 4, pp. 254-263, 2020. Article (CrossRef Link)

[29] D. Zou, F. Wang, N. Yu, and X. Kong, "Solving many-objective optimisation problems by an improved particle swarm optimisation approach and a normalised penalty method," International Journal of Bio-Inspired Computation, vol. 14, no. 4, pp. 247-264, 2019. Article (CrossRef Link)

[30] A. J. Nebro, J. J. Durillo, J. Garcia-Nieto, C. A. Coello, F. Luna, and E. Alba, "SMPSO: A new PSO-based metaheuristic for multi-objective optimization," in Proc. of Symposium on Computational intelligence in Miulti-criteria Decision-Making(MCDM), pp. 66-73, 2009. Article (CrossRef Link)

[31] E. Zitzler and L. Thiele, "Multiobjective evolutionary algorithms: a comparative case study and the strength Pareto approach," IEEE Transactions on Evolutionary Computation, vol. 3, no. 4, pp. 257-271, 1999. Article (CrossRef Link)

[32] L. While, L. Bradstreet, and L. Barone, "A Fast Way of Calculating Exact Hypervolumes," IEEE Transactions on Evolutionary Computation, vol. 16, no. 1, pp. 86-95, 2012. Article (CrossRef Link) 
[33] E. Zitzler, L. Thiele, M. Laumanns, C. M. Fonseca, and V. G. D. Fonseca, "Performance assessment of multiobjective optimizers: an analysis and review," IEEE Transactions on Evolutionary Computation, vol. 7, no. 2, pp. 117-132, 2003. Article (CrossRef Link)

[34] X. Cai, Z. Hu, and J. Chen, "A many-objective optimization recommendation algorithm based on knowledge mining," Information Sciences, vol. 537, pp. 148-161, 2020. Article (CrossRef Link)

[35] X. Zhang, Y. Tian, and Y. Jin, "A Knee Point-Driven Evolutionary Algorithm for Many-Objective Optimization," IEEE Transactions on Evolutionary Computation, vol. 19, no. 6, pp. 761-776, 2015. Article (CrossRef Link)

[36] N. A. Moubayed, A. Petrovski, and J. Mccall, "D2MOPSO: MOPSO Based on Decomposition and Dominance with Archiving Using Crowding Distance in Objective and Solution Spaces," Evolutionary Computation, vol. 22, no. 1, pp. 47-77, 2014. Article (CrossRef Link)

[37] T. Pamulapati, R. Mallipeddi, and P. N. Suganthan, "\$I_\{rm SDE $\}$ +-An Indicator for Multi and Many-Objective Optimization," IEEE Transactions on Evolutionary Computation, vol. 23, no. 2, pp. 346-352, 2019. Article (CrossRef Link)

[38] X. Cai, S. Geng, J. Zhang, D. Wu, Z. Cui, W. Zhang, and J. Chen, "A Sharding Scheme based Many-objective Optimization Algorithm for Enhancing Security in Blockchain-enabled Industrial Internet of Things," IEEE Transactions on Industrial Informatics, 2021. Article (CrossRef Link)
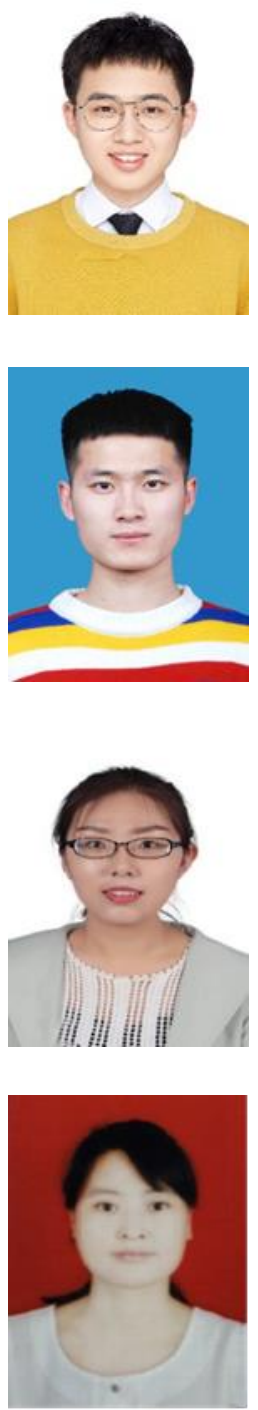

Zhaoming Hu is currently working toward M.S. degree at computer science and technology, Taiyuan University of Science and Technology, Taiyuan, China. His research interest includes bio-inspired computation and application.

Yang Lan is currently working toward M.S. degree at computer science and technology, Taiyuan University of Science and Technology, Taiyuan, China. His research interests include computational intelligence, combinatorial optimization and deep learning.

Zhixia Zhang is currently working toward Ph.D. degree at computer science and technology, Taiyuan University of Science and Technology, Taiyuan, China. Her research interest includes computational intelligence and network security.

Xingjuan Cai received her Ph.D. degree in Control Science and Engineering from Tongji University, China, in 2017. She is an Associate Professor with the school of Computer Science and Technology, Taiyuan University of Science and Technology, Taiyuan, China. Her research interest includes bio-inspired computation and application. 